\title{
Device for persons with low vision
}

\author{
Vlad Horațiu Sîrbu ${ }^{1, *}$, Daniela Mariana Barbu $^{1}$ \\ ${ }^{1}$ Transilvania University of Brasov, 29 Eroilor Bd., 500036 Brasov, Romania
}

\begin{abstract}
As defined by http://www.lowvision.org, "low vision is a bilateral impairment to vision that significantly impairs the functioning of the patient and cannot be adequately corrected with medical, surgical, therapy, conventional eyewear or contact lenses". The device is a keyboard that can be connected to any computer, phone or tablet via cable or Bluetooth. The keyboard will have eight buttons, two of them are predefined, and the other 6 form the Braille language cell.
\end{abstract}

\section{Introduction}

According to National Eye Institute (NEI) [1], low vision is a visual impairment that usually cannot be corrected by standard eyeglasses, contact lenses, medication, or surgery. It is often a loss of sharpness or acuity but may present as a loss of field of vision, light sensitivity, distorted vision or loss of contrast. Usual activities like reading, writing, watching $\mathrm{TV}$ or walking may be hard to do. Further, the mainly consequences of vision loss can cause anxiety, loneliness, helpless or even depressions of several kinds.

American Optometric Association consider low vision as a disorder caused by an ocular disease where the visual acuity is $20 / 200$ or less for the better eye and / or a field of vision of 20 degrees or less and can't be corrected or improved with regular glasses, surgery or medications [2].

The World Health Organization uses the following classifications of visual impairment [2]: viewing in the best eye with the best optical correction is:

- 20/20 to $20 / 60$, it is considered a slight loss of vision or an almost normal view;

- 20/70 to 20/160, is considered to be moderate visual impairment or low moderate vision;

- 20/200 to $20 / 400$, is considered a severe visual impairment or a severe low vision;

- $20 / 500$ to $20 / 1000$, is considered to be almost total visual impairment or almost total blindness;

- no perception of light, it is considered a total visual deficiency or total blindness or cecity.

The most common causes of low vision are [3] (figure 1):

- Macular degeneration - occurs due to circulatory disorders (which occur more often in the elderly) or factors not known primarily heredities (which we meet in children and young people).

- Diabetic retinopathy - occurs during diabetes and manifests as ophthalmic lesions (haemorrhages and white spots).

- Retinitis - it is a group of retinal lesions, due in part to circulatory disorders, plus a toxic factor.
- Amblyopia is a syndrome characterized by a decrease in visual acuity, whether or not associated with the modification of the foveal fixation reflex.

- Retinal detachment - in this disease, the retina separates from the underlying layer. This can be described as a curtain on one side of the field of vision.

- Cataracts - is the disorder that is manifested by the disorder of the lens that is subject to metabolic, physical, chemical, traumatic or age-related aggression.

- Glaucoma - under the name of glaucoma, there are a number of pathological conditions, the common symptom of which is the increase in eye pressure and the impairment of visual function.

- Acquired (traumatic injuries) - following a study, eye trauma can be of several kinds, namely: work accidents, accidents in everyday life, accident at birth, self-injury. Or, by their nature, trauma to the visual organ can be: contusions, actinic lesions, (physical or chemical), cuts cut or stung, with or without a foreign body.

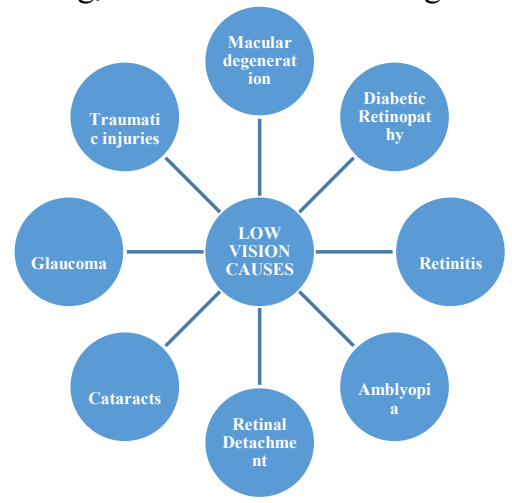

Fig. 1. Principal low vision causes

Some of these may be detected early and treatments can be made to stop disease progression (as macular degeneration or diabetes) or may have partial or total vision treatments such as retinal detachment or cataracts [4-5]. People with low vision may experience the following symptoms: loss of central vision, night blindness, loss of peripheral vision, blurred vision and hazy vision (figure 2) [6].

Email: sirbuvladhoratiu@gmail.com 


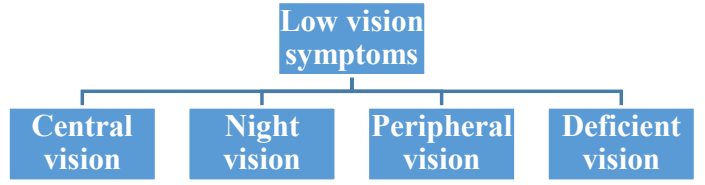

Fig. 2. Low vision symptoms

\section{Assisting systems for low vision and blindness}

\subsection{The cane}

This is the most distinctive sign of a man with a low vision or blindness. It is used for travel and is a very important tool in crossing the street because a person affected can cross a road without traffic signs only by lifting this cane [7]. There are several models available [8]:

- Long cane: is primarily designed as a mobility tool used to detect objects in a user's path. The length of the cane depends on the height of a user and extends traditionally from the floor to the user's sternum.

- Guide cane: This is a shorter cane that generally extends from the floor to the hip of the user - with a more limited mobility function. Used to scan borders and steps.

- Support cane: It is white and designed primarily to provide physical stability to a visually impaired user. Through its color, the cane also functions as a means of identification for someone with low vision or blindness.

- Green cane: Used in some countries to indicate a reduced vision, while the white cane indicates blindness.

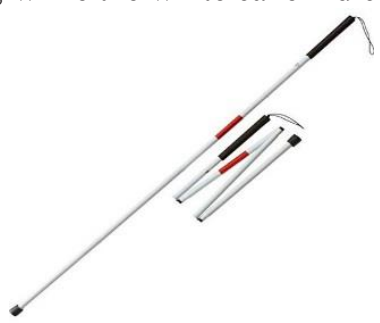

Fig. 3. The cane for blindness [8]

\subsection{Using Braille alphabet}

Braille is a write and print system for visual impairment people in which letters and numbers are identified by various arrangements of dots that are tactile [9]. It is a technique by which people with low or blindness can read and write. Each "cell" has 6 points, which are placed in a rectangle on 2 columns and 3 rows.

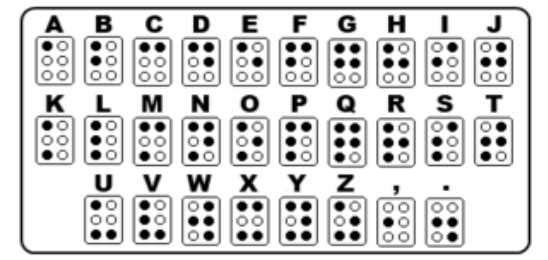

Fig. 4. Braille alphabet [9]

Braille can be "handwritten" - from right to left - with a paper puncher. When the sheet is inverted, the points are raised and the text can be read from left to right. It can also be achieved with the help of classical instruments, a slate and stylus with typewriters or access technology (Braille printers analogous to standard).

Therefore, the print / display equipment of Braille alphabet text, images, is based on a special paper that embosses the forms of the $3 \times 2$ matrix points that form the letters of the alphabet.

\subsection{Ocutech VES-AutoFocus-Glasses}

This device is an autofocus telescope, prescribed for the eye with a better sight [10]. It is mounted on special glasses frames, available in many colours and sizes. Also, the normal distance and bifocal prescription can also be included.

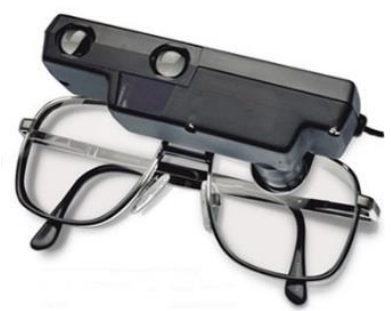

Fig. 5. Ocutech VES-AutoFocus [10]

VES-AutoFocus incorporates a 4x Keplerian telescope (the two lenses of the telescope are positive, the resulting image is real and reversed, prisms are used to reverse the image of the good field of view) coupled to an automated infrared computerized autofocus system [10]. It measures the focusing distance more than 30 times per sec to give a clear picture almost immediately and at any distance as close as 12 inches.

Operated with a separate, rechargeable battery, VESAutoFocus can be used for over 8 hours continuously. This method to reduce low vision was adopted by practitioners and people with visual impairments around the world.

\subsection{Smartwatch for the Blind}

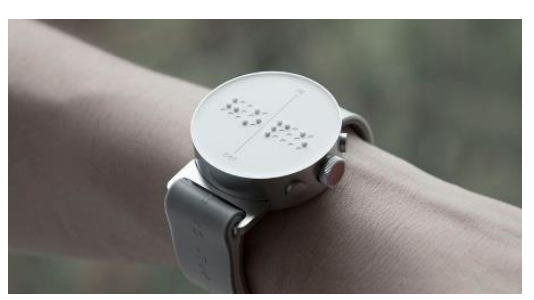

Fig. 6. Dot smartwatch [11]

Unlike most assisted smartwatches for blinds that rely on audio communication, Dot is the world's first Braille intelligent device that allows blind people to read messages on the screen. It has all the facilities a blind would need from a 21 st century device, such as social or email notifications, Google Maps instructions, or weather updates. All information is displayed on smartwatch using 4 Dynamic Braille cells and its users can select the speed at which characters are updated [11].

Just like any other smartwatch the Dot connects to a smartphone via Bluetooth, when connected it can receive any text information from any app [11]. 


\section{Device for persons with low vision}

This project is about creating a device for people with low vision or blindness.

Specifically, this device will be a simplified acquisition system that will act as a Braille keyboard. This will allow people with low vison and / or blind people to write various SMS, E-MAIL or even WORD documents without having to carry a large keyboard.

The device will simplify the lives of visually impaired people for whom the 21 st Century flat screens are not "communicative". The importance of the project is given by the constant evolution of devices for people with normal vision (from button phones to screen phones), a development that limits communication for people with low vision. Through this device, a new world opens for those with low vision to written communication and to what is "normal".

The first step in creating this project is designing the electrical system of the device, followed by a connection with Arduino Mega 2560 equipment and two keypads of four buttons each.

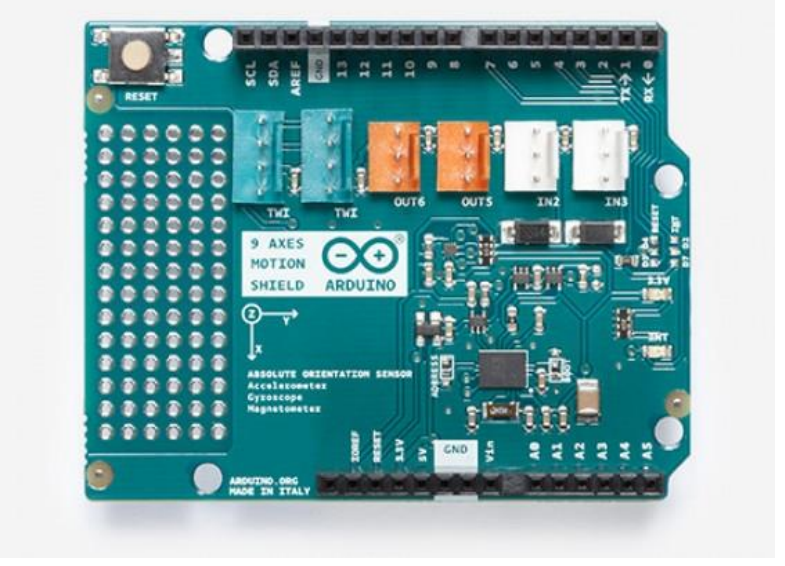

Fig. 6. Arduino Mega 2560 [12]

The MEGA 2560 is designed for more complex projects. With 54 digital I/O pins, 16 analogue inputs and a larger space for your sketch it is the recommended board for 3D printers and robotics projects. This gives your projects plenty of room and opportunities [12].

This keypad has 4 buttons, and since every key has its own wire line, no matrix code is required - just treat these like every day switches. The membrane is soft and has a removable paper backing to expose a strong adhesive so you can stick this on an enclosure and feed the cable through a slot.

The software will be loaded onto the board and tested for functionality. The results will be displayed on a LCD screen and transmitted through Bluetooth, Wi-Fi or cable.

The two keyboards each with 4 buttons will consist of a Braille cell (this consists of two columns of three buttons), and the other two buttons will have predefined space and delete functions [13].

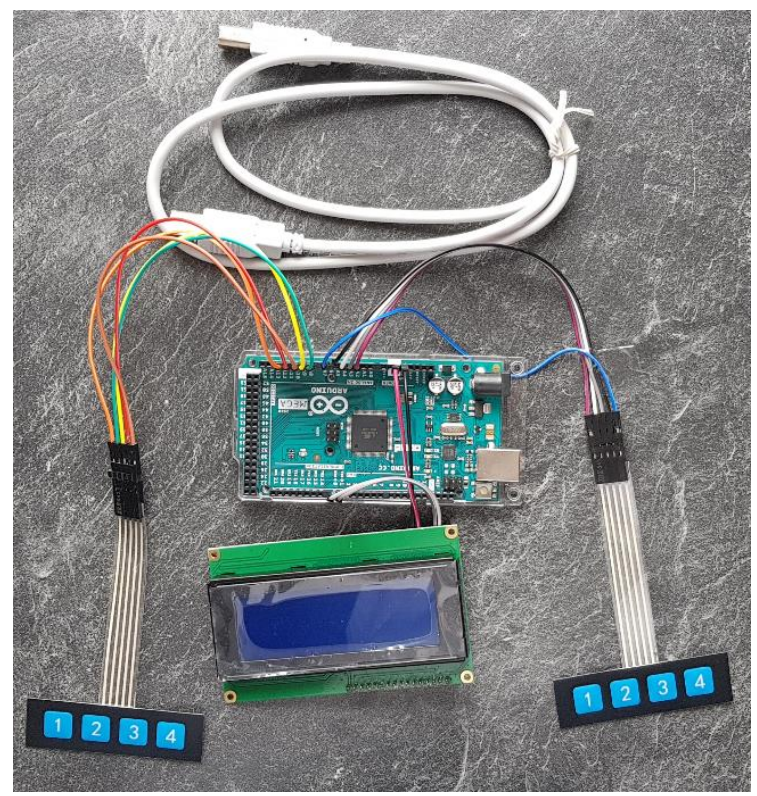

Fig. 7. Electronic components

The second step is designing the case with „Computeraided design" (CAD) software and then to be produced from plastic (PLA) with a Creality 3D printer.

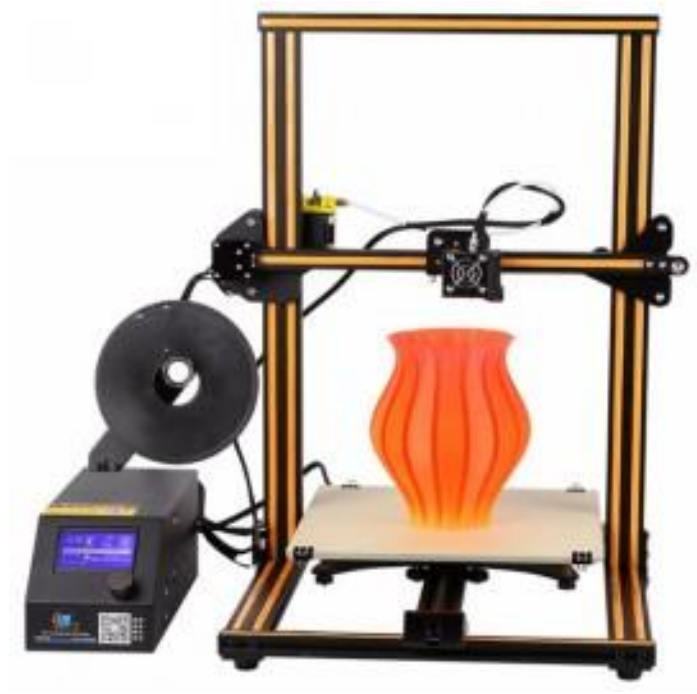

Fig. 8. Creality 3D CR-10S-3 [14]

This is an open-frame printer and heated print pad that can use a wide range of thermoplastic materials including PLA, ABS, TPU, Copper PET and composite materials [14].

Important specifications:

- High accuracy

- Print speed max. $200 \mathrm{~mm} / \mathrm{s}$

- Max. Print pad temperature $60^{\circ} \mathrm{C}$

- Layer thickness 0.05-0.35 mm

- Extrusion temperature max. $250^{\circ} \mathrm{C}$ 


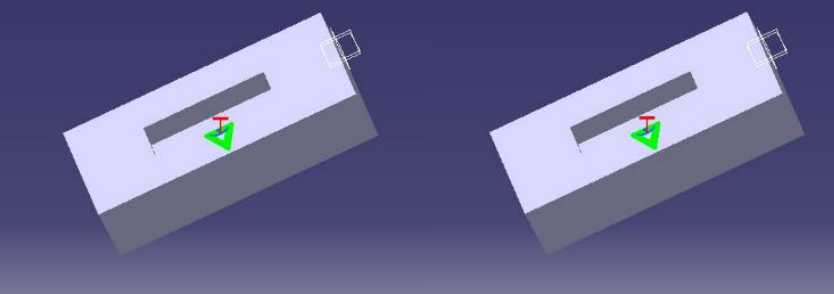

Fig. 9. CAD prototype of the case

Before the actual printing, the CAD model will be introduced into software called Cura 3.2.1., where the necessary print settings will be made.

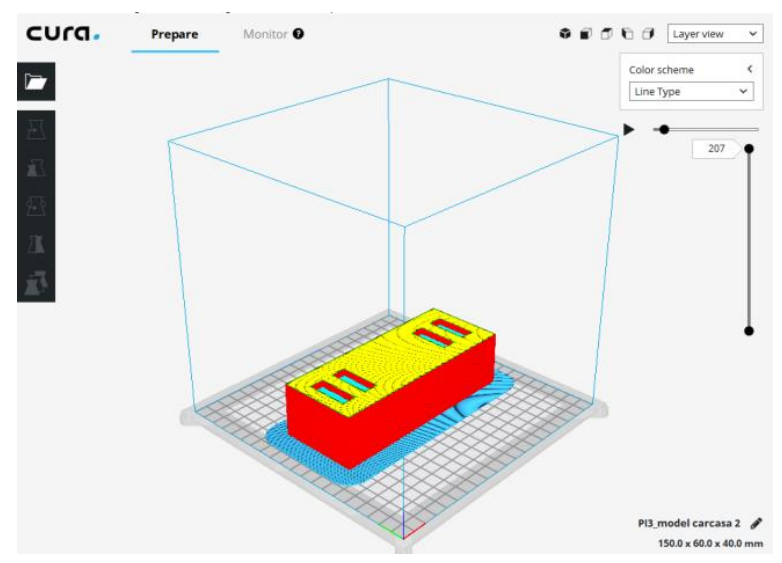

Fig. 10. Preparation of the casing printing and related settings

The final device will be assembled from the electrical part and the casing specially designed to implement all the necessary components.

The construction of the system has certain risks, such as applying over voltages on certain electrical components and implicitly damaging them, or creating multiple carcases in order to get the most ergonomic option, but these risks are part of testing a prototype, which means that broken components will be replaced and are assumed.

Once the system has been fully assembled, tests will be performed to verify that the device returns the desired characters. A series of instructions for commissioning and using the device will be prepared

\section{Conclusions}

In conclusion, this keypad prototype designed for people with low vision is designed to improve their communication capabilities by writing with technology.
With its small size, lightweight materials, the device will be easy to carry and handle which opens their world to SMS, E-mails and is a step towards a common communication in the century of devices.

One of the main goals of the project is the low cost of the final product, because many devices designed for the visually impaired people have high prices which is why not many people use them.

This study was funded through a winning project in a university competition for final study projects.

\section{References}

1. Official Site of National Eye Institute (NEI) https://nei.nih.gov/

2. Official Site of American Optometric Association, https://www.aoa.org/documents/optometrists/CPG14.pdf

3. STL Chung, IL Bailey, G Dagnelie, et al., Optometry and vision science: official publication of the American Academy of Optometry, 89(9), 1244-1245 (2012)

4. HR Taylor, Investigative Ophthalmology \& Visual Science, 44,1413-1418 (2003)

5. MI Baritz, Cyberpsychology \& Behavior 8(4), 304 305 (2005)

6. EM Vingolo, $\mathrm{V}$ De Rosa, D Domanico, $\mathrm{F}$ Anselmucci, Clinical Optometry, 7, 53-58 (2015)

7. QK Dang, Y Chee, DD Pham, YS Suh, Sensors (Basel, Switzerland); 16(1), 95 (2016)

8. SM Bradley, CR Hernandez, American Family Physician. 84 (4) (2011)

9. J Jiménez, et al., Survey of Ophthalmology, 54(1), 142-149 (2009)

10. Official Site of Ocutech, https://www.ocutech.com/product/ocutech-vesautofocus/

11. Official Site of Steemit, https://steemit.com/technology/@penguinpablo/thedot-world-s-first-braille-smartwatch-for-the-blind

12. Official Site of Arduino, https://store.arduino.cc/arduino-mega-2560-rev3

13. Official Site of Adafruit, https://www.adafruit.com/product/1332

14. Official Site of Formwerk, http://formwerk.ro/ro imprimante.html 